\title{
Magnetic Field Effect on Topological Spin Excitations in $\mathrm{CrI}_{3}$
}

\author{
Lebing Chen $\odot,{ }^{1}$ Jae-Ho Chung $\odot,{ }^{2, *}$ Matthew B. Stone $\odot,{ }^{3}$ Alexander I. Kolesnikov $\odot,{ }^{3}$ Barry Winn, ${ }^{3}$ V. Ovidiu Garlea, ${ }^{3}$ \\ Douglas L. Abernathy $\odot,{ }^{3}$ Bin Gao $\odot,{ }^{1}$ Mathias Augustin, ${ }^{4,6}$ Elton J. G. Santos, ${ }^{4,5,6, \dagger}$ and Pengcheng Dai $\circledast^{1, \$}$ \\ ${ }^{1}$ Department of Physics and Astronomy, Rice University, Houston, Texas 77005, USA \\ ${ }^{2}$ Department of Physics, Korea University, Seoul 02841, Korea \\ ${ }^{3}$ Neutron Scattering Division, Oak Ridge National Laboratory, Oak Ridge, Tennessee 37831, USA \\ ${ }^{4}$ Institute for Condensed Matter Physics and Complex Systems, School of Physics and Astronomy, \\ The University of Edinburgh, EH9 3FD, United Kingdom \\ ${ }^{5}$ Higgs Centre for Theoretical Physics, The University of Edinburgh, EH9 3FD, United Kingdom \\ ${ }^{6}$ Donostia International Physics Center, Paseo Manuel de Lardizabal 4, 20018, San Sebastian, Spain
}

(Received 18 February 2021; revised 6 May 2021; accepted 10 June 2021; published 31 August 2021)

The search for topological spin excitations in recently discovered two-dimensional (2D) van der Waals $(\mathrm{vdW})$ magnetic materials is important because of their potential applications in dissipationless spintronics. In the $2 \mathrm{D}$ vdW ferromagnetic (FM) honeycomb lattice $\mathrm{CrI}_{3}\left(T_{C}=61 \mathrm{~K}\right)$, acoustic and optical spin waves are found to be separated by a gap at the Dirac points. The presence of such a gap is a signature of topological spin excitations if it arises from the next-nearest-neighbor (NNN) Dzyaloshinskii-Moriya (DM) or bond-angle-dependent Kitaev interactions within the $\mathrm{Cr}$ honeycomb lattice. Alternatively, the gap is suggested to arise from an electron correlation effect not associated with topological spin excitations. Here, we use inelastic neutron scattering to conclusively demonstrate that the Kitaev interactions and electron correlation effects cannot describe spin waves, Dirac gaps, and their in-plane magnetic field dependence. Our results support the idea that the DM interactions are the microscopic origin of the observed Dirac gap. Moreover, we find that the nearest-neighbor (NN) magnetic exchange interactions along the $c$ axis are antiferromagnetic (AF), and the NNN interactions are FM. Therefore, our results unveil the origin of the observed $c$-axis $\mathrm{AF}$ order in thin layers of $\mathrm{CrI}_{3}$, firmly determine the microscopic spin interactions in bulk $\mathrm{CrI}_{3}$, and provide a new understanding of topology-driven spin excitations in $2 \mathrm{D} \mathrm{vdW}$ magnets.

DOI: 10.1103/PhysRevX.11.031047

\section{INTRODUCTION}

The discovery of robust two-dimensional (2D) ferromagnetic (FM) long-range order in monolayer van der Waals $(\mathrm{vdW})$ magnets $[1-3]$ is important because these materials can provide a new platform to study fundamental physics without the influence of a substrate and can potentially be used to develop new spintronic devices [4,5]. One prominent group of these materials includes the chromium trihalides, $\mathrm{CrX}_{3}(\mathrm{X}=\mathrm{Br}, \mathrm{I})$ or $\mathrm{CrXTe}_{3}(\mathrm{X}=\mathrm{Ge}, \mathrm{Si})$, where $\mathrm{Cr}^{3+}$ $\left(3 d^{3}, S=3 / 2\right)$ ions form 2D honeycomb lattices [Fig. 1(a)] $[6,7]$. Within a single honeycomb layer, $\mathrm{Cr}^{3+}$ ions interact with each other ferromagnetically via the nearly 90-degree

\footnotetext{
jaehc@korea.ac.kr †esantos@exseed.ed.ac.uk *pdai@rice.edu
}

Published by the American Physical Society under the terms of the Creative Commons Attribution 4.0 International license. Further distribution of this work must maintain attribution to the author(s) and the published article's title, journal citation, and DOI.
Subject Areas: Condensed Matter Physics, Spintronics, Topological Insulators
Cr-X-Cr superexchange paths [Fig. 1(b)] [8]. Although the $3 d$ electrons of $\mathrm{Cr}^{3+}$ do not provide large spin-orbit coupling (SOC), the heavier ligand atoms such as iodine may serve as a source of significant SOC. This not only provides the thermal stability observed in vdW layered materials but also enriches the physics of magnetism in the 2D limit [9-14]. Indeed, it is proposed that the Kitaev interaction [15], known to be important for effective $S=1 / 2$ honeycomb lattice magnets near a Kitaev quantum spin liquid [16,17], may occur in $S=3 / 2 \mathrm{CrI}_{3}$ across the nearest bond with bonddependent anisotropic Ising-like exchange [Fig. 1(b)]. This occurrence would be critical for the magnetic stability of monolayer $\mathrm{CrI}_{3}$ and spin dynamics in bulk $\mathrm{CrI}_{3}$ [18-22]. Furthermore, spin waves (magnons) from honeycomb ferromagnets can be topological by opening a gap at the Dirac points via time-reversal symmetry breaking (TRSB) $[23,24]$. As a magnetic analog of electronic dispersion in graphene [25], spin-wave spectra of honeycomb ferromagnets have Dirac points at the Brillouin zone boundaries where dispersions of acoustic and optical spin waves meet and produce Dirac cones. If the system has TRSB arising from a large SOC, one would expect to observe an energy gap at 
the Dirac point of the bulk magnon bands [23], analogous to the SOC-induced gap at the Dirac point in the electronic dispersion of graphene [26]. This energy gap, in turn, would allow the realization of massless topological spin excitations propagating without dissipation [27-29].

Experimentally, a spin gap was indeed observed at the Dirac point in the spin-wave spectra of the honeycomb lattice $\mathrm{FM} \mathrm{CrI}_{3}$ [30]. Three possible scenarios have been proposed to understand the observed spin gap. The first corresponds to the Dzyaloshinskii-Moriya (DM) interaction that occurs on the bonds without inversion symmetry [Figs. 1(a), 1(c), and 1(d)] [31,32]. The second scenario is the Kitaev interaction that also breaks time-reversal symmetry and can inhabit nontrivial topological edge modes $[19,33]$. Finally, the observed Dirac spin gap is suggested to arise from electron correlations that must be treated explicitly to understand the spin dynamics in $\mathrm{CrI}_{3}$ and the broad family of 2D vdW magnetic materials [34]. In this case, spin excitations in $\mathrm{CrI}_{3}$ would not be topological.

Another intriguing property of $\mathrm{CrI}_{3}$ is its weak structural and magnetic coupling along the $c$ axis. In the lowtemperature $\mathrm{FM}$ phase, bulk $\mathrm{CrI}_{3}$ is assumed to have rhombohedral lattice structure with space group $R \overline{3}$ [6]. On warming across $T_{C}$, the $\mathrm{FM}$ order in $\mathrm{CrI}_{3}$ disappears in a weakly first-order phase transition coupled with a small

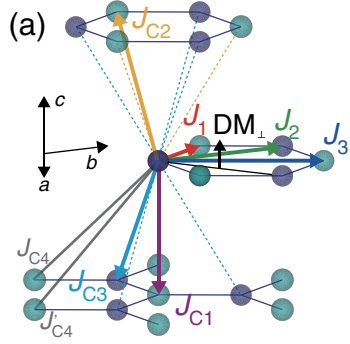

(c)

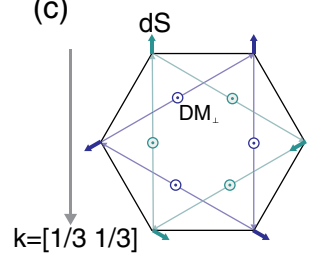

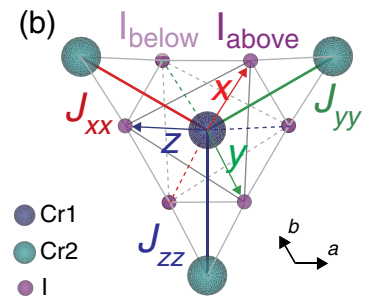

(d)

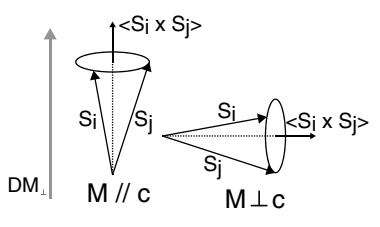

FIG. 1. Crystal structure of $\mathrm{CrI}_{3}$. (a) $\mathrm{CrI}_{3}$ rhombohedral lattice showing only $\mathrm{Cr}$ atoms, with $\mathrm{Cr}^{3+}$ spins along the $c$ axis. $\mathrm{Cr} 1$ (blue) and $\mathrm{Cr} 2$ (cyan) spheres indicate $\mathrm{Cr}$ atoms in different triangular sublattices. The colored bonds indicate in-plane and interlayer magnetic exchange interactions. The cyan and yellow dashed lines show the three $J_{c 2}$ 's and six $J_{c 3}$ 's around one $\mathrm{Cr}$ atom. (b) Kitaev interaction in the local coordinates of $\mathrm{CrI}_{3}$. The $J_{x x}, J_{y y}, J_{z z}$ bond is between the NNs, and the $\{x, y, z\}$ direction is parallel to the Cr-I bond, as shown with arrows. (c) DM interactions in $\mathrm{CrI}_{3}$ with a top view of the $\mathrm{Cr}^{3+}$ hexagon at the Dirac wave vector. The cyan and blue colors distinguish two triangular sublattices. (d) Interactions between DM and spins. Only when spins have components along the $c$ axis can the DM term give a nonzero contribution to the total Hamiltonian. $c$ axis lattice parameter change. Upon further warming to 90-200 K, $\mathrm{CrI}_{3}$ undergoes a first-order phase transition from rhombohedral to monoclinic structure with a $C / 2 m$ space group, basically shifting the stacking of the $\mathrm{CrI}_{3}$ layers [6]. From comparisons to spin-wave dispersions, the nearest-neighbor (NN) $c$ axis magnetic exchange coupling is deduced to be FM with $J_{c 1} \approx 0.59 \mathrm{meV}$ [Fig. 1(a)] [30]. However, transport, Raman scattering, scanning magnetic circular dichroism microscopy, and tunneling measurements as a function of film thickness $[1,35,36]$, pressure [37,38], and applied magnetic field [39] suggest $A$-type antiferromagnetic (AF) structure associated with the monoclinic structure present in the bilayer and a few top layers of bulk $\mathrm{CrI}_{3}$. In particular, a magnetic field a few Tesla along the $c$ axis was found to modify the crystal lattice symmetry of $\mathrm{CrI}_{3}$, thus suggesting a strong spin-lattice coupling [39]. Therefore, it is important to determine if the NN interlayer exchange coupling is indeed FM and what determines the overall FM interlayer coupling in the $\mathrm{CrI}_{3}$ bulk with rhombohedral lattice structure.

In this work, we use high-resolution inelastic neutron scattering to study spin waves of $\mathrm{CrI}_{3}$ and their magnetic field dependence. By reducing the mosaic of coaligned single crystals of $\mathrm{CrI}_{3}$ from earlier work [30], we were able to precisely measure the magnitude of the spin gap at the Dirac points and the entire spin-wave spectra. In addition, we determine the effect of an in-plane magnetic field on spin waves and the Dirac spin gap in $\mathrm{CrI}_{3}$. By comparing the experimental observations with expectations from the Heisenberg-DM and Heisenberg-Kitaev Hamiltonian, and the effect of electron correlations, we conclude that spin waves and the Dirac spin gap in $\mathrm{CrI}_{3}$ cannot be described by the Heisenberg-Kitaev Hamiltonian and electron correlation effects. Instead, the data are approximately consistent with the Heisenberg-DM Hamiltonian, considering both the $c$-axis and in-plane DM interactions. Our results therefore clarify the microscopic spin interactions in $\mathrm{CrI}_{3}$ and provide a new understanding of topology-driven spin excitations in $2 \mathrm{D} v \mathrm{vW}$ magnets.

\section{RESULTS}

Single crystalline $\mathrm{CrI}_{3}$ samples were grown using the chemical-vapor-transport method as described in Ref. [6]. Our inelastic neutron scattering experiments were carried out on either fully coaligned $(\sim 0.42 \mathrm{~g})$ or $c$-axis aligned $(\sim 1 \mathrm{~g})$ crystals on the SEQUOIA [40], HYSPEC [41], and ARCS [42] spectrometers at Spallation Neutron Source, Oak Ridge National Laboratory. Consistent with Ref. [30], we use a honeycomb lattice with an in-plane $\mathrm{Cr}$-Cr distance of about $3.96 \AA$ and $c$ axis layer spacing of $6.62 \AA$ in the low-temperature rhombohedral structure to describe $\mathrm{CrI}_{3}$. The momentum transfer $\mathbf{Q}=H \mathbf{a}^{*}+K \mathbf{b}^{*}+L \mathbf{c}^{*}$ is denoted as $(H, K, L)$ in reciprocal lattice units (r.l.u.) with marked high-symmetry points [Figs. 2(a) and 2(b)]. All measurements were carried out with the $c$ axis of the 

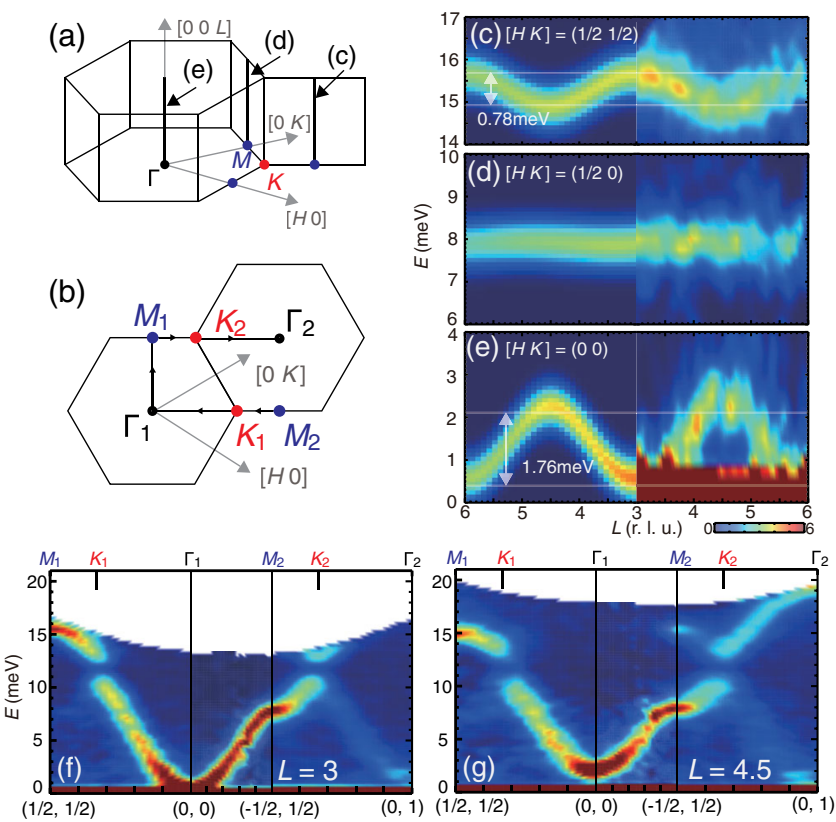

FIG. 2. Spin wave spectra of $\mathrm{CrI}_{3}$. (a) The hexagonal reciprocal lattice of $\mathrm{CrI}_{3}$. Gray arrows show reciprocal lattice vectors, and high-symmetry $(\Gamma, K, M)$ points are specified in blue $(M)$, red $(K)$, and black $(\Gamma)$ dots, respectively. The bold black lines specify the scan direction in (c-e). (b) Projection of the hexagonal reciprocal lattice in the $[H, K]$ plane. The arrows indicate the scan path of the spectra shown in (f, g), Figs. 3(a)-3(c), and Figs. 4(a) and 4(b). (c-e) The spin wave dispersion along the $L$ direction at different $[H, K]$ positions specified in (a), showing different bandwidths at different $[H, K]$ points. The left and right panels are calculation and data, respectively. (f, g) Spin wave dispersion at different $L$ points. (f) shows $L$ integration range $[2.5,3.5]$ near the $[0,0, L]$ band bottom, while $(\mathrm{g})$ shows $L$ integration range $[4,5]$ near the band top.

sample in the horizontal scattering plane and with the applied magnetic fields vertical, i.e., in the $a b$ plane of $\mathrm{CrI}_{3}$ [Figs. 1(a), (c), and (d)].

We begin by describing the zero field high-resolution spinwave data of $\mathrm{CrI}_{3}$ obtained on SEQUIOA (Figs. 2 and 3). Figure 3(a) shows the energy-momentum $(E-\mathbf{Q})$-dependent spin-wave spectra along the high-symmetry directions in reciprocal space as depicted in Fig. 2(a). These in-plane spinwave spectra were obtained by integrating dispersive spin waves along the $c$ axis over $-5 \leq L \leq 5$. The overall momentum dependence of the spin-wave energies is consistent with previous work [30], revealing two spin-wave modes characteristic of the honeycomb ferromagnets. The lower and upper modes account for the acoustic and optical vibrations, respectively, of the two sublattice spins. These two spin-wave modes will meet each other at the Dirac wave vectors of $Q_{K_{1}}=\left(\frac{1}{3}, \frac{1}{3}\right)$ and $Q_{K_{2}}=\left(\frac{2}{3},-\frac{1}{3}\right)$ [Figs. 3(a) and 3(d)]. Inspection of Fig. 3(a) reveals clear evidence of a spin gap of about $2.8 \mathrm{meV}$, which is approximately $50 \%$ the value estimated from previous low-resolution data [30].
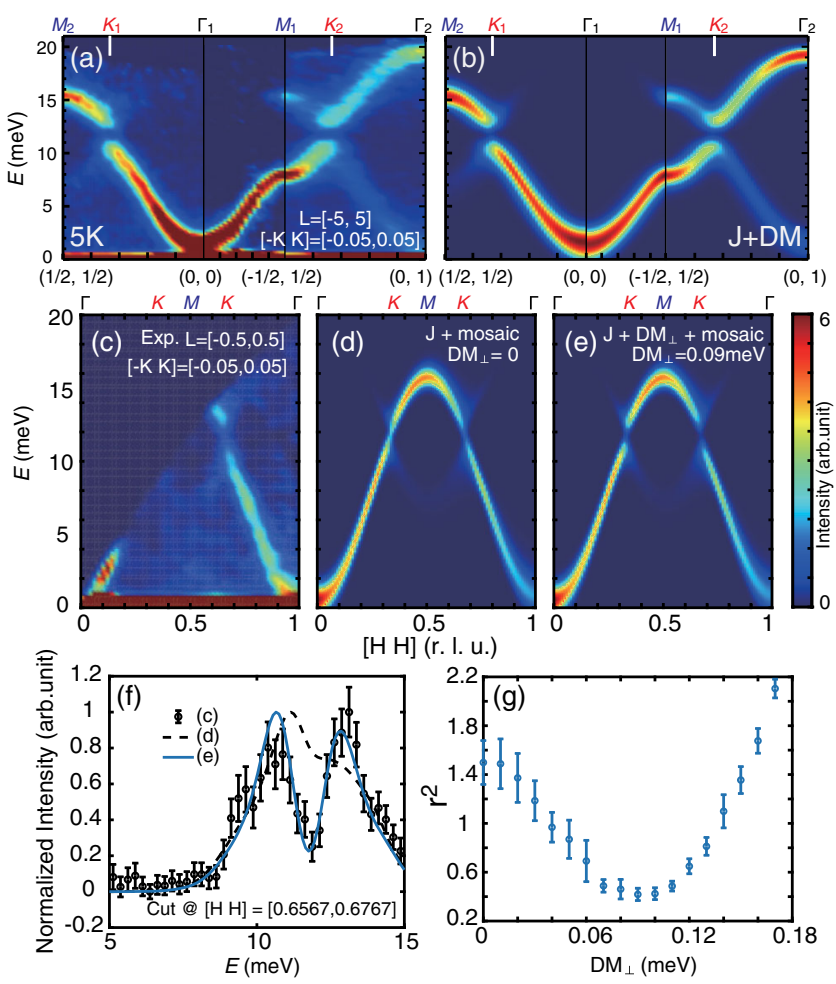

FIG. 3. The Heisenberg-DM model fit of $\mathrm{CrI}_{3} E-\mathbf{Q}$ spin wave spectrum. High-symmetry points are labeled. (a) Experimental data at $5 \mathrm{~K}$. (b) Heisenberg-DM model simulation using parameters in Table I. (c) Experimental data at $5 \mathrm{~K}$ with smaller $L$ integration range. (d, e) Heisenberg-DM model simulations including sample mosaic with (d) $\mathrm{DM}=0$ and (e) $\mathrm{DM}_{\perp}=$ $0.09 \mathrm{meV}$. (f) Constant-Q cuts of the data in (c-e) at the Dirac point $(2 / 3,2 / 3,0)$. (g) The squared error $r^{2}$ between experimental and simulation values of the Dirac point cut as a function of the DM interaction strength.

This result is mostly due to the reduced mosaicity of the coaligned single crystals (an in-plane mosaic full width at half maximum of $8.0^{\circ}$ compared with that of about $17^{\circ}$ in Ref. [30]) and improved instrumental resolution [43].

To completely determine the spin-wave spectra of $\mathrm{CrI}_{3}$, we show in Figs. 2(c)-2(e) the $L$ dependence of spin waves at different in-plane wave vectors. Inspection of the figures reveals that the modes along the $\left[\frac{1}{2}, \frac{1}{2}, L\right]$ and $[0,0, L]$ directions exhibit mutually opposite $L$ dependence. Along the $[0,0, L]$ direction, the spin-wave dispersion exhibits a minimum of $0.4 \mathrm{meV}$ at $L=3 n(n=$ integers $)$ and a maximum of $2.1 \mathrm{meV}$ at $L=3 n+\frac{3}{2}$ [Fig. 2(e)]. In contrast, the mode along the $\left[\frac{1}{2}, \frac{1}{2}, L\right]$ direction peaks at $L=3 n$ and has a minimum at $L=3 n+\frac{3}{2}$ [Fig. 2(c)], while spin waves along the $\left[\frac{1}{2}, 0, L\right]$ direction are featureless [Fig. 2(d)]. The overall spin-wave spectra at $L=3$ and 4.5 are shown in Figs. 2(f) and 2(g), respectively. The opposite $L$ dependence between the high- and low-energy spin waves requires finite FM interplane exchanges along the bonds that are tilted off the $c$ axis. 
To understand spin-wave spectra in Figs. 2 and 3, we consider a Heisenberg model with the DM interaction to account for the observed Dirac spin gap [23,24,30]. The Hamiltonian of the DM interaction, $H_{\mathrm{DM}}$, can be written as $H_{\mathrm{DM}}=-\sum_{i<j}\left[\mathbf{A}_{i j} \cdot\left(\mathbf{S}_{i} \times \mathbf{S}_{j}\right)\right]$, where $\mathbf{S}_{i}$ and $\mathbf{S}_{j}$ are spins at sites $i$ and $j$, respectively, and $\mathbf{A}_{i j}$ is the antisymmetric DM interaction between sites $i$ and $j$ [Figs. 1(a) and 1(c)]. The combined Heisenberg-DM (J-DM) Hamiltonian is $H_{\mathrm{J}-\mathrm{DM}}=\sum_{i<j}\left[J_{i j} \mathbf{S}_{i} \cdot \mathbf{S}_{j}+\mathbf{A}_{i j} \cdot \mathbf{S}_{i} \times \mathbf{S}_{j}\right]+\sum_{j} D_{z}\left(S_{j}^{z}\right)^{2}$, where $J_{i j}$ is the magnetic exchange coupling of the $\mathbf{S}_{i}$ and $\mathbf{S}_{j}$, and $D_{z}$ is the easy-axis anisotropy along the $z(c)$ axis [30]. As shown in Fig. 1(a), we define the in-plane NN, the next-nearest-neighbor ( $\mathrm{NNN}$ ), and the third $\mathrm{NN}$ interactions as $J_{1}, J_{2}$, and $J_{3}$, respectively. The $c$ axis $\mathrm{NN}$, the NNNs, and the third $\mathrm{NN}$ interactions are $J_{c 1}, J_{c 2} / J_{c 3}$, and $J_{c 4} / J_{c 4}^{\prime}$, respectively. For ideal honeycomb lattice materials where the NNN bond breaks the inversion symmetry [Fig. 1(c)], the DM vectors can have both inplane $\left(\mathrm{DM}_{\|}\right)$and out-of-plane $\left(\mathrm{DM}_{\perp}\right)$ components, but the former will not contribute to the topological gap opening because of the threefold rotational symmetry of the honeycomb lattice [Fig. 1(d)]. As a result, only the DM term parallel to the $c$ axis, i.e., the NNN DM interaction, will contribute to the opening of a spin gap in spin-wave spectra. Since bulk $\mathrm{CrI}_{3}$ orders ferromagnetically below a Curie temperature of $T_{C} \approx 61 \mathrm{~K}$ with an ordered moment along the $c$ axis [6], one can fit the spin-wave spectra and Dirac gap using the finite NNN $H_{\mathrm{DM}}(\neq 0)$, which may induce TRSB and topological spin excitations in the FM ordered state [30].

The left panels of Figs. 2(c)-2(e) and 3(b) are the calculated spin-wave spectra with exchange parameters listed in Table I [30]. Given the nearly flat dispersion along the $\left[\frac{1}{2}, 0, L\right]$ direction shown in Fig. 2(d), we choose to set $J_{c 2}=J_{c 3}$ for the two interplane NNN exchanges of nearly identical bond lengths [Fig. 1(a)]. The best-fit parameters reveal that the $\mathrm{NN}$ interlayer magnetic interactions are AF with strong FM couplings along the NNN directions [Fig. 1(a)]. In addition, one must include a finite DM interaction $A$ to account for the observed spin gap at the Dirac points [Figs. 3(a), 3(b), and 3(d)] [43]. To precisely determine the magnitude of $A$, we consider spin wave data at the Dirac point with a narrow $c$ axis integration range of $-0.5 \leq L \leq 0.5$ in Fig. 3(c). Figures 3(d) and 3(e) show calculated spin wave spectra taking into account the mosaic of the aligned single crystals of $\mathrm{CrI}_{3}$ without and with the NNN DM interactions, respectively. An energy cut through Dirac point reveals clearly that the calculated spectra with the NNN DM interaction fits the data better [Fig. 3(f)]. The best magnitude of $A\left(\mathrm{DM}_{\perp}\right)$ is determined by the least squares method using the observed and calculated spin wave spectra [Fig. 3(g)]. Since the Dirac wave vector is along the zigzag bonds of the honeycomb lattice, the observation of a spin gap at the Dirac point indicates a
TABLE I. Magnetic exchange interaction strength (the negative value indicates the FM exchange) in the J-DM model, the electron correlation model, and the J-K- $\Gamma$ model. Our estimated $\mathrm{DM}_{\perp} \approx 0.09 \mathrm{meV}$ is similar to that in Ref. [44].

\begin{tabular}{lccc}
\hline \hline Model & $\mathrm{J}-\mathrm{DM}$ & $\mathrm{J}_{\mathrm{c} 4}$ & $\mathrm{~J}-\mathrm{K}-\Gamma$ \\
\hline$J_{1}(\mathrm{meV})$ & -2.11 & -2.11 & -0.83 \\
$J_{2}(\mathrm{meV})$ & -0.11 & -0.11 & -0.16 \\
$J_{3}(\mathrm{meV})$ & 0.10 & 0.10 & 0.08 \\
$J_{c 1}(\mathrm{meV})$ & 0.048 & 0.048 & 0.048 \\
$J_{c 2}\left(J_{c 3}\right)(\mathrm{meV})$ & -0.071 & -0.071 & -0.071 \\
$J_{c 4}\left(-J_{c 4}^{\prime}\right)(\mathrm{meV})$ & 0 & -0.1 & 0 \\
$\mathrm{DM}_{\perp}(\mathrm{meV})$ & 0.09 & 0 & 0 \\
$K(\mathrm{meV})$ & 0 & 0 & -3.8 \\
$D_{z}(\mathrm{meV})$ & -0.123 & -0.123 & 0 \\
$\Gamma(\mathrm{meV})$ & 0 & 0 & -0.082 \\
\hline \hline
\end{tabular}

symmetry-breaking field between the two $\mathrm{Cr}$ sublattices within the honeycomb lattice [Figs. 1(a) and 1(c)]. While the DM vectors may be oriented either along the $c$ axis or perpendicular to it, only the $c$ axis component can open the Dirac gap due to the threefold symmetry of the ideal honeycomb lattice. In addition, the magnitude of the gap is directly proportional to the $c$ axis component of the ordered spins $[44,45]$.

An alternative scenario to understand the observed spin gap at the Dirac point is through the Kitaev interaction that occurs across the nearest bond with bond-dependent anisotropic Ising-like exchange [Fig. 1(b)] [15], which also breaks the time-reversal symmetry and can inhabit nontrivial topological edge modes [19]. The Kitaev interaction Hamiltonian $H_{K}$ is $H_{K}=\sum_{\langle i j\rangle \in \lambda \mu(\nu)}\left[K S_{i}^{\nu} S_{j}^{\nu}+\right.$ $\left.\Gamma\left(S_{i}^{\lambda} S_{j}^{\mu}+S_{i}^{\nu} S_{j}^{\lambda}\right)\right]$, where $(\lambda, \mu, \nu)$ are any permutation of $(x, y, z), K$ is the strength of the Kitaev interaction, and $\Gamma$ is the symmetric off-diagonal anisotropy that induces a spin gap at the $\Gamma$ point $[19,33]$. The combined HeisenbergKitaev Hamiltonian, the so-called J-K- $\Gamma$ Hamiltonian, is $H_{\mathrm{J}-\mathrm{K}-\Gamma}=\sum_{\langle i j\rangle \in \lambda \mu(\nu)}\left[J_{i j} \mathbf{S}_{i} \cdot \mathbf{S}_{j}+K S_{i}^{\nu} S_{j}^{\nu}+\Gamma\left(S_{i}^{\lambda} S_{j}^{\mu}+S_{i}^{\nu} S_{j}^{\lambda}\right)\right]$. By fitting the J-K- $\Gamma$ Hamiltonian using the data shown in Fig. 3(a), we extract the exchange parameters shown in Table I, whose Kitaev term $(-3.8 \mathrm{meV})$ is smaller than that of Ref. [33] (-5.6 meV) due to the smaller energy gap observed from better aligned samples. When FM ordered spins are oriented along the $c$ axis [6], the spin Hamiltonian based on the Heisenberg-Kitaev exchanges can also reproduce the observed spin waves and energy gap at the Dirac point in $\mathrm{CrI}_{3}$ [33]. Therefore, one cannot determine whether the NNN DM or Kitaev model is responsible for the spin gap at Dirac points in the spin waves of $\mathrm{CrI}_{3}$ at zero field [33].

Finally, by using calculations beyond density functional theory (DFT), it was suggested that the observed Dirac spin gap arises from the electron correlations not considered in the usual DFT theory [34]. In this picture, the Dirac spin gap arises from the differences in $c$ axis magnetic exchange 

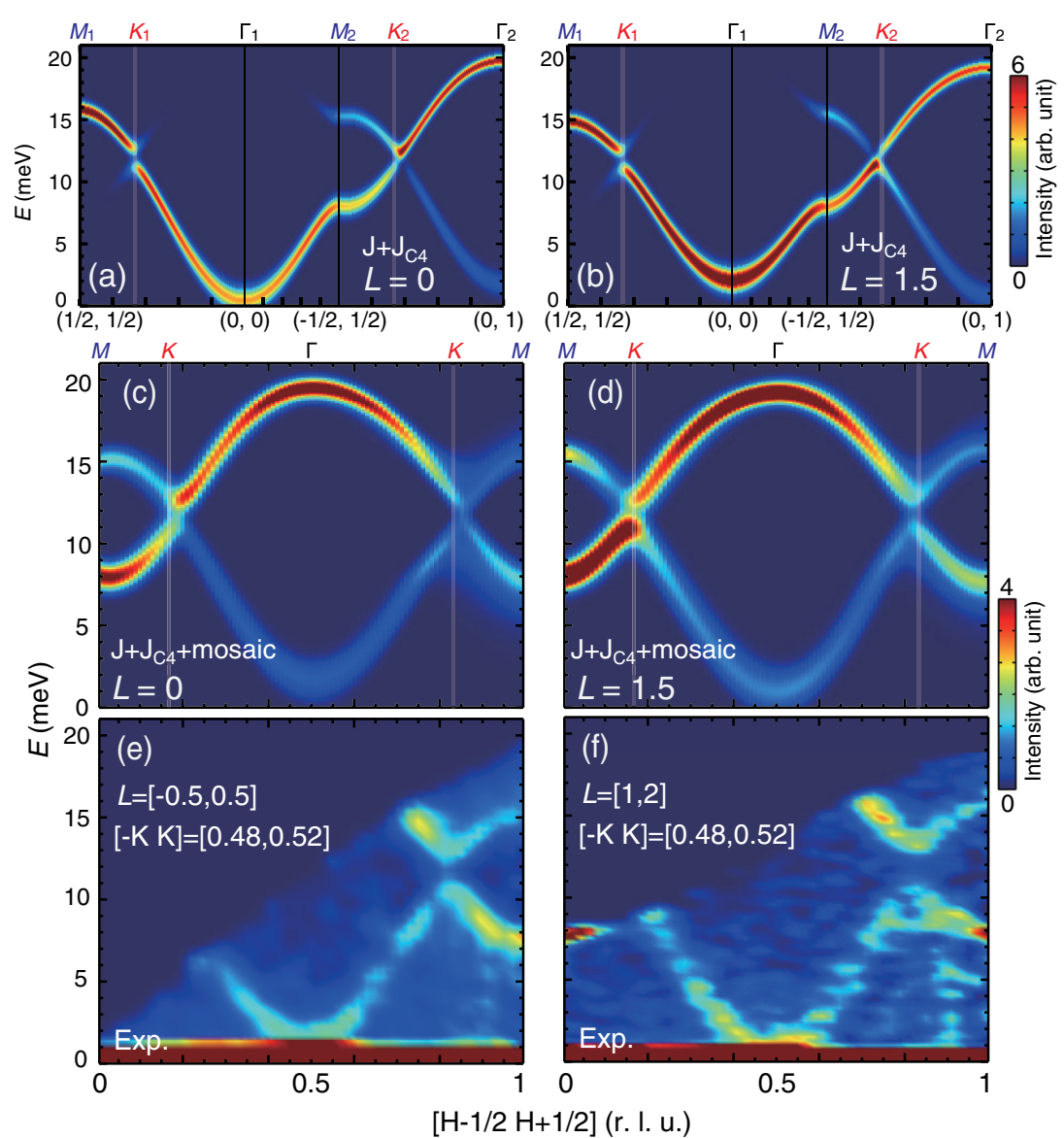

FIG. 4. The Heisenberg- $J_{c 4}$ (spin correlation) model simulation of $\mathrm{CrI}_{3}$ spin wave spectra, here $J_{c 4}=-J_{c 4}^{\prime}=-0.10 \mathrm{meV}$. (a, b) Inplane spin excitation spectrum with $L=0$ and 1.5, respectively. (c, d) Spin excitation spectra along (c) $[H-1 / 2, H+1 / 2,0]$ and (d) $[H-1 / 2, H+1 / 2,1.5]$ directions, respectively. The integration and mosaic effects are considered in the plot. (e, f) Experimental data corresponding to (c) and (d), respectively. The integration range of (c-f) are specified in (e) and (f).

pathways along the third $\mathrm{NN}_{c 4}$ and $J_{c 4}^{\prime}$ [Fig. 1(a), and see Fig. 3 in Ref. [34] ]. If this picture is correct, one would expect that Dirac nodal lines, where acoustic and optical spin-wave bands cross, wind around the Dirac $K$ point along the $L$ direction [34]. Since both $J_{c 4}$ and $J_{c 4}^{\prime}$ connect with $\mathrm{Cr} 1$ and do not break the $\mathrm{CrI}_{3}$ sublattice symmetry, the electron correlation effects do not produce a true Dirac spin gap and only cause the Dirac crossing to shift sideways and induce nodal winding along the $c$ axis. The spin-wave intensity winding around the Dirac point has been observed in the insulating easy-plane honeycomb quantum magnet $\mathrm{CoTiO}_{3}$ without a Dirac spin gap and DM interaction, suggesting the nontrivial topology of the Dirac magnon wave functions [46-49].

Figures 4(a) and 4(b) show expected spin-wave spectra at $L=0$ and 1.5 , respectively, calculated using a Heisenberg Hamiltonian with magnetic exchange parameters specified in Table I. Near the Dirac points, we see spin gaplike features at $K_{1}$ and $K_{2}$ due to shifted acousticoptical spin-wave touching points, and there is no true spin gap near the Dirac points. To compare with experimental observations, we calculate spin waves with the sample mosaic in Figures 4(c) and 4(d), which broaden spin waves but do not change their basic characters. Since experimental data at these $L$ values show a clean spin gap at all wave vectors near the Dirac points [Figs. 4(e) and 4(f)]. We conclude that the observed Dirac spin gap cannot arise from the electron correlation effects as discussed in Ref. [34].

Since both the NNN DM and Kitaev models can describe spin waves of $\mathrm{CrI}_{3}$ [33], it will be important to determine which microscopic model is correct. One way to separate these two scenarios is to do an inelastic neutron scattering experiment on $\mathrm{CrI}_{3}$ with a magnetic field applied within the $a b$ plane. The easy axis of spins in $\mathrm{CrI}_{3}$ is parallel to the $c$ axis, but a magnetic field of $3 \mathrm{~T}$ will turn the spin to the $a b$ plane with almost zero out-of-plane components [6]. This change of the FM ordered moment direction will nullify the NNN DM term by making $\mathbf{A}_{i j}$ and $\mathbf{S}_{i} \times \mathbf{S}_{j}$ perpendicular to each other with vanishing $H_{\mathrm{DM}}$, and therefore close the NNN DM interaction-induced spin gap at the Dirac points [Fig. 1(d)]. This result is similar to the 2D kagome lattice ferromagnet $\mathrm{Cu}[1,3-$ benzenedicarboxylate (bdc)] [Cu(1,3-bdc)], where an out-of-plane magnetic field applied to align the in-plane FM ordered moments along the $c$ axis is found to also induce a DM interaction-induced 

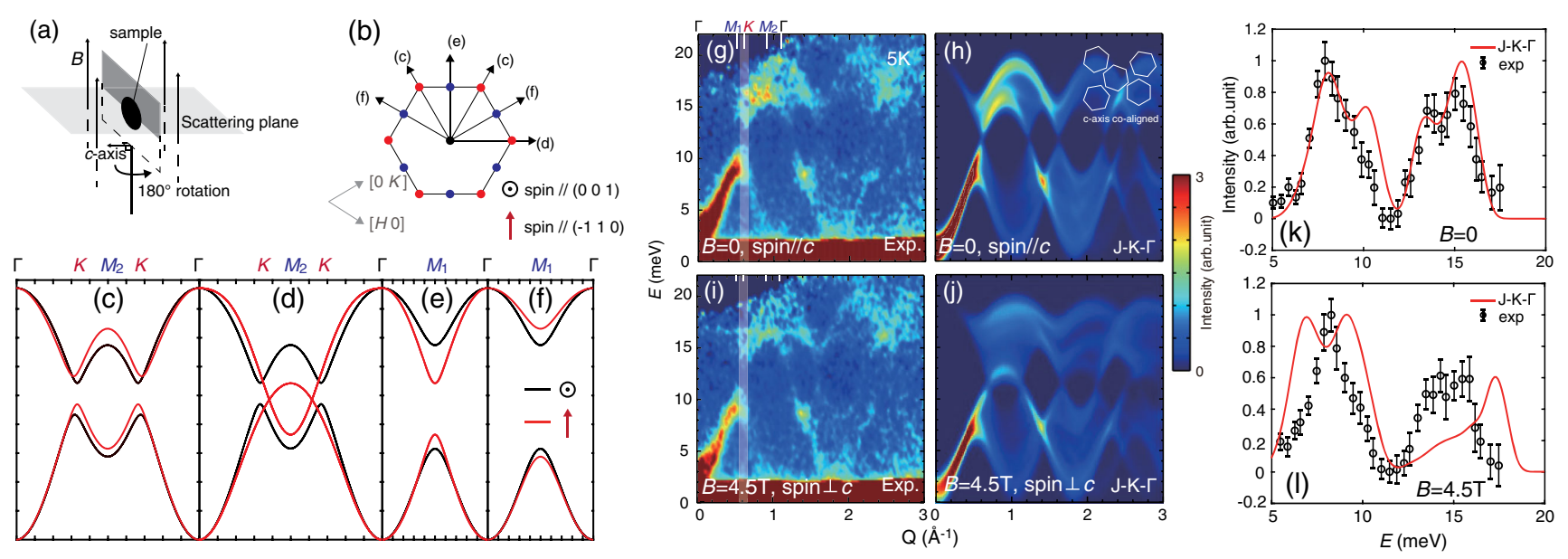

FIG. 5. The in-plane magnetic field effects on spin waves of $c$ axis aligned $\mathrm{CrI}_{3}$ single crystals shown in the inset of (h), and Heisenberg-Kitaev model fit of the spectra. (a) The experimental setup of inelastic neutron scattering experiments, where applied field is vertical and $c$ axis of the crystals is in the light-shaded horizontal scattering plane. (b) The reciprocal lattice showing the scan direction in $(\mathrm{c}-\mathrm{f})$. The high symmetry points are shown with blue $(M)$, red $(K)$, and black $(\Gamma)$ dots. (c-f) Spin wave dispersions of the HeisenbergKitaev model with in-plane (red) and out-of-plane (black) spin orientations. (g, i) Spin wave $E$-Q spectra of $\mathrm{CrI}_{3}$ at $5 \mathrm{~K}$ in zero and $4.5 \mathrm{~T}$ in-plane fields, respectively. The high-symmetry points are marked on top. Here $\mathbf{Q}$ in the unit of $\AA^{1}$ indicates the wave vector $\mathrm{s}$ projection on the $[H, K]$ plane with $L=[-5,5]$ integration. (h, j) Calculated $E$-Q $\mathbf{Q}$ spectra using the Heisenberg-Kitaev Hamiltonian at zero and $4.5 \mathrm{~T}$ field, respectively. (k, l) Comparison of the energy cuts between experiments (black dots) and calculations (red lines) using the Heisenberg-Kitaev Hamiltonian at Dirac point in $0 \mathrm{~T}$ and $4.5 \mathrm{~T}$, respectively. The $\mathbf{Q}$ integration range of the energy cuts is $0.55-0.66 \AA^{1}$ centered around the $K$ point $\left(=0.608 \AA^{1}\right)$, as shown in the long white shaded line in (g) and (i).

spin gap at the Dirac points [50,51]. In contrast, if the spin gap at the Dirac point is induced by the Kitaev exchange, its field dependence will be anisotropic and dependent on the relative angle of the polarized spin with respect to the inplane lattice orientation [Figs. 5(a)-5(f)].

To test this idea, we perform inelastic neutron scattering experiments under in-plane magnetic fields on HYSPEC [41] with an incident neutron energy of $E_{i}=27 \mathrm{meV}$ (Fig. 5) and on ARCS [42] with $E_{i}=23 \mathrm{meV}$ (Fig. 6). Figure 5(a) shows the geometry of the experimental setups, where the applied magnetic fields are vertical in the honeycomb lattice plane. For HYSPEC experiments, we use $c$ axis aligned single crystals $(\sim 1 \mathrm{~g})$ [see inset of Fig. 5(h)] and apply a field of $4.5 \mathrm{~T}$, which is larger than the in-plane saturation field of $3 \mathrm{~T} \mathrm{[6]}$ and sufficient to completely polarize the moment in the $\mathrm{CrI}_{3}$ plane. As a function of increasing field, the spin gap at the $\Gamma$ point $(\approx 0.4 \mathrm{meV})[33]$ initially decreases to overcome the $c$ axis aligned moment but then increases because of the increasing Zeeman energy [43]. These results are consistent with the field dependence of the gap from either singleion spin anisotropy or the off-diagonal $\Gamma$ term in the Kitaev interaction [43].

Figures 5(g) and 5(i) show the spin-wave Q- $E$ spectra at zero and 4.5-T field, respectively. While the overall spinwave intensity decreases at $4.5 \mathrm{~T}$ because of the rotation of the spin moment direction from the $c$ axis to the $\mathrm{CrI}_{3}$ plane, the spin gap near the Dirac point, marked by the white vertical line in Figs. 5(g) and 5(i), shows no obvious change. In the J-K- $\Gamma$ model, the spin gap opens at the Dirac points because the NN Kitaev exchange interactions alternate between two different anisotropic bond-dependent terms along the zigzag bonds [19]. Since the Kitaev interaction Hamiltonian $H_{K}$ is inherently sensitive to the spin orientations, spin-wave spectra of a $\mathrm{J}-\mathrm{K}-\Gamma$ model will change drastically when the moment direction of the spins is rotated from the $c$ axis to the in-plane direction by an externally applied magnetic field [Figs. 5(b)-5(f)]. Whereas a DM interaction-induced spin gap would close uniformly under an in-plane field to preserve the sixfold inplane symmetry of the spin-wave dispersion, the Kitaev interaction-induced spin gaps will respond anisotropically depending on the relative angles between the wave vector and field direction. Furthermore, the field-induced changes in spin-wave spectra will not be limited around the Dirac points in the J-K- $\Gamma$ model.

Figures 5(h) and 5(j) show calculated spin-wave Q- $E$ spectra using the J-K- $\Gamma$ Hamiltonian with the $c$ axis and inplane moment, respectively. We use the exchange parameters that reproduce the zero field spectra identically with the Heisenberg-DM model shown in Fig. 3(b) [33]. While the zero field calculation agrees well with the data, the 4.5-T spin-wave spectra are clearly different from that of the calculation. The data points in Figs. 5(k) and 5(l) show energy-dependent spin waves across the Dirac point at 0 and 4.5 $\mathrm{T}$, respectively. The solid lines are spin-wave 

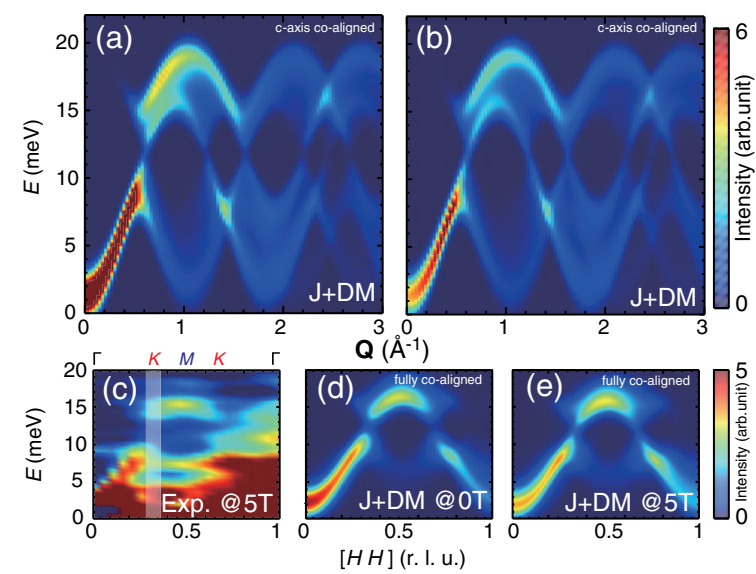

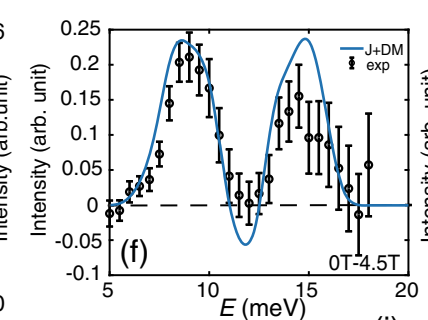

(h)
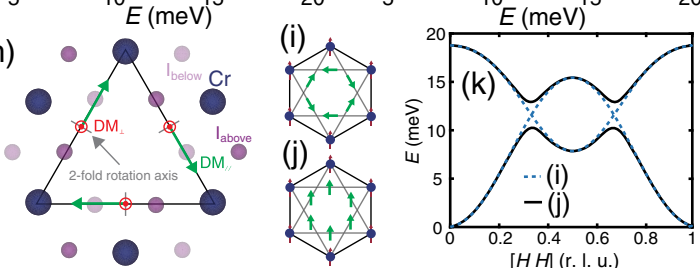

FIG. 6. The magnetic field effect on the Heisenberg-DM model. (a, b) Calculated $E$ - $\mathbf{Q}$ spectra of $c$ axis aligned $\mathrm{CrI}_{3}$ using the Heisenberg-DM Hamiltonian with 0 and $4.5 \mathrm{~T}$ in-plane fields, respectively. (c) Spin waves of a fully co-aligned $\mathrm{CrI}_{3}$ single crystals near the Dirac point along the $[H, H]$ direction with a $5 \mathrm{~T}$ in-plane magnetic field. (d, e) Heisenberg-DM model simulation with 0 and 5 T inplane field, respectively. (f) The effect of a magnetic field on spin wave dispersion near Dirac point and its comparison with the Heisenberg-DM calculations. (g) Constant-Q cut at the Dirac point $([H, H]=(0.3,0.37)$ ) on the experimental data and Monte Carlo simulations. The experimental data has a constant background subtracted. The gray dots show intensity increasing due to higher instrumental background. (h) Schematics of the in-plane DM interaction of a triangular sublattice in one Cr hexagon. The in-plane component of the DM interaction $\left(\mathrm{DM}_{\|}\right)$is perpendicular to the two-fold rotation axis between the two NN Cr ions according to the Moriya s rule. (i) In-plane DM interactions respecting the three-fold symmetry of the lattice. (j) An example of in-plane DM interactions breaking the three-fold symmetry of the lattice. $(\mathrm{k})$ The calculation of spin wave dispersion with in-plane spins and in-plane DM interactions shown in red dashed lines (i) and black solid lines (j). Here $\mathrm{DM}_{\|}=0.17 \mathrm{meV}$.

calculations using the $\mathrm{J}-\mathrm{K}-\Gamma$ Hamiltonian with the $c$ axis and in-plane moments, confirming that the HeisenbergKitaev Hamiltonian clearly fails to describe the magnetic field effect on spin waves.

Figures 6(a) and 6(b) show calculated spin-wave $\mathbf{Q}-E$ spectra using the Heisenberg-DM Hamiltonian with the $c$ axis and in-plane moment, respectively. Compared with the J-K- $\Gamma$ Hamiltonian in Figs. 5(h) and 5(j), the HeisenbergDM Hamiltonian obviously agrees much better with the experimental data in Figs. 5(g) and 5(i). Figure 6(c) shows the $\mathbf{Q}-E$ dependence of spin waves near the Dirac point with an in-plane applied field of $5.0 \mathrm{~T}$ at $5 \mathrm{~K}$, obtained on coaligned single crystals of $\mathrm{CrI}_{3}$ on ARCS. Figures 6(d) and 6(e) are the corresponding spin-wave spectra calculated using the Heisenberg-DM Hamiltonian. The data points in Fig. 6(f) show the magnetic field difference plot obtained from Figs. 5(k) and 5(1). It is clear that the solid line calculated from the Heisenberg-DM Hamiltonian can approximately describe the data but with a small deviation near the Dirac point [Figs. 6(f) and 6(g)].

From the above discussions, we see that the J-K- $\Gamma$ Hamiltonian clearly cannot describe the observed magnetic field dependence of spin waves in $\mathrm{CrI}_{3}$. While the simple NNN Heisenberg-DM Hamiltonian can describe the overall spectra and its magnetic field dependence, it may have difficulty in describing the magnetic field dependence of the Dirac spin gap. Since the loss of translational symmetry between the two $\mathrm{Cr}$ sublattice spins of an ideal honeycomb lattice can open a spin gap at the Dirac points, it is important to determine other possible origins for the observed Dirac gap.

\section{DISCUSSION}

In previous work [1,35-39], $A$-type $\mathrm{AF}$ order of $\mathrm{CrI}_{3}$ was found to be associated with the monoclinic structural phase either near the surface of the bulk or in thin-layer form (for example, the bilayer of $\mathrm{CrI}_{3}$ ). However, it is unclear why the $\mathrm{AF}$ order in bilayer $\mathrm{CrI}_{3}$ has monoclinic crystal structure, which appears in the paramagnetic phase above $T_{C}$ of bulk $\mathrm{CrI}_{3}[1,35,36]$. Using the NN AF and

TABLE II. Estimated magnetic bonding energies associated with each $\mathrm{Cr}^{3+}$ atom in various crystal structure and exchange couplings [43]. Rhom and mono indicate rhombohedral and monoclinic lattice structures, respectively. In the hypothetical mono-bulk and mono-bilayer cases, the NN and NNN magnetic exchange couplings are assumed to be the same as those of the rhom-bulk and rhom-bilayer, revealing that the FM rhombohedral lattice structure has lower magnetic bonding energy.

\begin{tabular}{lccc}
\hline \hline Structures & $J_{c 1}(\mathrm{meV})$ & $J_{c 2}(\mathrm{meV})$ & Energy $(\mathrm{meV})$ \\
\hline Rhom-bulk, FM & 0.048 & -0.071 & -1.33 \\
Rhom-bilayer, FM & 0.048 & -0.071 & -0.66 \\
Mono-bulk, FM & 0.048 & -0.071 & -0.21 \\
Mono-bilayer, FM & 0.048 & -0.071 & -0.10 \\
Mono-bulk, AF & 0.037 & 0 & -0.33 \\
Mono-bilayer, AF & 0.037 & 0 & -0.17 \\
\hline \hline
\end{tabular}


NNN FM interlayer coupling in the rhombohedral FM phase (Figs. 1 and 2), we estimate that the interlayer stacking is still FM in the bilayer limit [43], thus ruling out rhombohedral AF bilayer structure. If we change the crystal structure to monoclinic but maintain the NN and NNN $c$ axis coupling in bulk $\mathrm{CrI}_{3}$, the magnetic bonding energies are higher than that of the rhombohedral lattice structure. From Raman scattering of bilayer $\mathrm{CrI}_{3}$, the sum of the interlayer AF coupling in monoclinic structure was found to be about $0.11 \mathrm{meV}$ [36]. Assuming that the NNN magnetic exchange is negligible, we estimate that the NN magnetic exchange in the monoclinic bilayer is $J_{c 1}=0.037 \mathrm{meV}$ [43]. Table II summarizes the total magnetic bonding energy for one $\mathrm{Cr}^{3+}$ atom in different lattice and magnetic structures [43]. We find that the FM bilayer rhombohedral structure should be more favorable than the AF bilayer monoclinic structure, contrary to the observation. Since Raman experiments can only deduce total magnetic exchange along the $c$ axis, we are unable to determine the actual NN and NNN magnetic exchange couplings in the monoclinic structure. Nevertheless, the observed AF order in the monoclinic bilayer suggests that such a phase has lower ground-state energy compared with that of the FM rhombohedral structure in bulk or bilayer $\mathrm{CrI}_{3}$. As the hydrostatic pressure applied on the AF bilayer $\mathrm{CrI}_{3}$ can reduce the interlayer spacing and reintroduce the rhombohedral FM state [38], we expect that the monoclinic bilayer $\mathrm{CrI}_{3}$ should have a larger $c$-axis $\mathrm{AF}$ exchange and lattice parameter compared with that of the rhombohedral bilayer. This case is also consistent with a reduced $c$-axis lattice constant below $T_{C}$ in bulk $\mathrm{CrI}_{3}$ [6] and recent simulations of transport measurements suggesting that the layers may expand along the $c$ axis to minimize interaction energy and stabilize a different magnetic coupling [52,53]. We note that the collinear AF order in iron pnictides also expands the lattice parameter along the AF ordering direction $[54,55]$. While the NNN interlayer exchange couplings of bulk $\mathrm{CrI}_{3}$ ultimately determines its FM ground state, the AF interlayer coupling prevails in the monoclinic bilayer $\mathrm{CrI}_{3}$ [43]. These results suggest that the monoclinic-torhombohedral structural phase transition in $\mathrm{CrI}_{3}$ is driven by reducing the interlayer magnetic exchange energy.

Although our data rule out a pure Kitaev interaction and electron correlations as the microscopic origins of the observed Dirac spin gap, there may be other interactions in addition to the NNN DM that contribute to the Dirac spin gap. We consider several possibilities. First, reducing the bulk structural symmetry from rhombohedral to monoclinic, by itself, will not open a spin gap at the Dirac point because such a structural phase transition does not change the inversion symmetry of the $\mathrm{Cr}$ honeycomb sublattice. If additional structural deformations are present due to, for instance, thermal effects, the inversion center between the first NNs would be removed, which, incidentally, would allow DM interactions to exist at that level. Nevertheless, we show in the Supplemental Material [43] that the inclusion of DM at the first NNs does not open a gap at the Dirac point. We also consider a Heisenberg model with both the NNN DM and Kitaev interaction [43]. By using a Heisenberg-DM Hamiltonian with different Kitaev interaction strengths that fits spin-wave spectra at $0 \mathrm{~T}$, we can compare the expected and observed spin waves under a 4.5-T field and in-plane spin. The result indicates that the Kitaev term should be near zero in order to get the best fit to the 4.5-T spin-wave spectra [43].

Alternatively, magnon-magnon interactions may potentially affect $H_{\mathrm{DM}}$, which can result in a gap at the Dirac point. When higher-order Holstein-Primakoff transformations are considered in the description of the spin interactions in $\mathrm{CrI}_{3}$, three-operator products arise which may contribute to the gap [43]. However, since magnon-magnon interactions in most magnetic materials are weakly energy and wave vector dependent, and typically occur at energies above the single magnon scattering, they are unlikely to give rise to the observed spin gap at the Dirac points.

Finally, we envision two mechanisms that may allow the spin gap at the Dirac point to remain open under an in-plane spin-polarizing field: The first is the sublattice symmetry breaking, and the second is the threefold rotational symmetry breaking of the ideal honeycomb lattice of $\mathrm{CrI}_{3}$.

We first discuss the possible sublattice symmetry breaking of an ideal honeycomb lattice. From spin-wave spectra in Figs. 2 and 3, we know that the two $\mathrm{Cr}^{3+}$ ions of different sublattices within the honeycomb unit cell interact not only via the intralayer $\mathrm{NN}$ interaction $J_{1}$ but also the interlayer $\mathrm{NN}_{c 1}$, which is AF and directly along the $c$ axis [Fig. 1(a)]. Whereas both bonds are bisected by the structural inversion centers, respectively, the interlayer AF exchange coupling $J_{c 1}$ will favor a breaking of the inversion symmetry between the two $\mathrm{Cr}$ sublattice spins. As a result, if the two $\mathrm{Cr}^{3+}$ ions within a unit cell have spins of unequal moments (due to environmental defects such as $\mathrm{Cr}$ and/or I vacancy) [56], an energy gap will appear at the Dirac points without significantly affecting spin waves at other wave vectors.

It is well known that the interlayer magnetic order in $\mathrm{CrI}_{3}$ switches from AF to FM as the number of stacked vdW layers increases from the bilayer to the bulk, accompanied by a structural transition from monoclinic to rhombohedral stacking along the $c$ axis [57-62]. In addition, a small $(<3 \mathrm{~T})$ in-plane magnetic field can easily transform $\mathrm{AF}$ ordered multilayer $\mathrm{CrI}_{3}$ into a ferromagnet [63]. Even in the bulk samples, the surface layers are reported to have $\mathrm{AF}$ monoclinic structure that can be tuned by a $c$-axis aligned magnetic field of a few Tesla [39]. While these results indicate minor energy differences in rhombohedral and monoclinic structures of $\mathrm{CrI}_{3}$, they suggest that the $\mathrm{Cr}$ honeycomb lattice may have subtle NN inversion symmetry-breaking structural distortions that are responsible for the observed Dirac spin gap [56]. 
We next consider the field-induced breaking of the threefold symmetry of the in-plane DM vectors. Since the NNN DM interaction must involve the iodine atoms, the mirror symmetry of the simple honeycomb lattice is lost, with only the twofold rotation axis remaining [43]. As a result, the DM vector is not constrained to be out of plane and can have in-plane projections. This argument holds as long as the DM vector is perpendicular to the twofold rotation axis according to Moriya's rule [Fig. 6(h)]. In the case where no magnetic field is applied, the spins are aligned along the $c$ axis, and only the DM vector component parallel to this direction can open the Dirac gap. In the situation where an in-plane applied magnetic field is strong enough to rotate the $c$ axis aligned spins into the $\mathrm{CrI}_{3}$ plane, the threefold symmetry of the in-plane DM vectors will cancel out when determining the spin-wave energy at the $K$ point, thus yielding no contribution to the Dirac gap [Figs. 6(i) and 6(j)]. However, if the in-plane DM vector breaking the threefold symmetry is induced by the applied field, then it will contribute to opening a Dirac gap [Fig. 6(k)]. This process will require a significant fieldinduced symmetry breaking of the in-plane DM whose energy scale should be similar to the out-of-plane DM terms $(\sim 0.17 \mathrm{meV})$. While a $c$ axis aligned magnetic field of a few Tesla is known to break the lattice symmetry of $\mathrm{CrI}_{3}$ [39], there is currently no direct experimental proof that an in-plane magnetic field of a few Tesla would break the threefold symmetry of the crystalline lattice in $\mathrm{CrI}_{3}$. Nevertheless, we could estimate a band gap of $\sim 1.1 \mathrm{meV}$ using the parameters extracted from our data, which underestimates the gap value obtained from the experiments due to the mosaicity effect [43]. This conjecture suggests that the $\mathrm{Cr}$ lattice, as well as its halide sublattice, contributes to the topological spin features observed in $\mathrm{CrI}_{3}$.

\section{CONCLUSIONS}

In summary, we used inelastic neutron scattering to study the impact of an in-plane magnetic field on spin waves of $\mathrm{CrI}_{3}$. At zero field, we completely determined the magnetic exchange couplings along the $c$ axis by carefully measuring $c$-axis spin-wave dispersions at different in-plane wave vectors. We find that the $\mathrm{NN} c$-axis magnetic exchange coupling is AF and the NNN magnetic exchange couplings are FM. These results thus indicate coexisting AF and FM exchange interactions between the hexagonal layers of $\mathrm{CrI}_{3}$. We also confirmed the presence of a spin gap at the Dirac points at zero field and found that an in-plane magnetic field that can rotate the moment from the $c$ axis to the $\mathrm{CrI}_{3}$ plane also modifies the spin-wave spectra and spin gap at Dirac points. These results can conclusively rule out the $\mathrm{J}-\mathrm{K}-\Gamma$ Hamiltonian and electron correlations as origins of the Dirac spin gap. While the field dependence of the Dirac spin gap may not be completely understood within the NNN Heisenberg-DM Hamiltonian, the results suggest the presence of a local sublattice or threefold rotational symmetry breaking of the ideal honeycomb lattice in $\mathrm{CrI}_{3}$. Our results therefore firmly establish the microscopic spin Hamiltonian in $\mathrm{CrI}_{3}$ and provide a new understanding of topology-driven spin excitations in 2D vdW magnets.

\section{ACKNOWLEDGMENTS}

We are grateful to Franz G. Utermohlen, Nandini Trivedi, Adam Tsen, Zurab Guguchia, Liuyan Zhao, and Rui He for helpful discussions. The neutron scattering and sample growth work at Rice is supported by U.S. NSFDMR-1700081 and by the Robert A. Welch Foundation under Grant No. C-1839 (P. D.). The work of J. H. C. was supported by the National Research Foundation (NRF) of Korea (Grants No. 2020R1A5A1016518 and No. 2020K1A3A7A09077712). E. J. G. S. acknowledges computational resources through the CIRRUS Tier-2 HPC Service (ec131 CirrusProject) at EPCC funded by the University of Edinburgh and EPSRC (EP/P020267/1); and ARCHER UK National Supercomputing Service via Project d429. E. J.G.S. acknowledges the Spanish Ministry of Science's grant program "EuropaExcelencia" under Grant No. EUR2020-112238, EPSRC Early Career Fellowship (EP/T021578/1), and the University of Edinburgh for funding support. A portion of this research used resources at the Spallation Neutron Source, a DOE Office of Science User Facility operated by the Oak Ridge National Laboratory.

[1] B. Huang et al., Layer-Dependent Ferromagnetism in a van der Waals Crystal down to the Monolayer Limit, Nature (London) 546, 270 (2017).

[2] C. Gong et al., Discovery of Intrinsic Ferromagnetism in Two-Dimensional van der Waals Crystals, Nature (London) 546, 265 (2017).

[3] Z. Zhang, J. Shang, C. Jiang, A. Rasmita, W. Gao, and T. Yu, Direct Photoluminescence Probing of Ferromagnetism in Monolayer Two-Dimensional $\mathrm{CrBr}_{3}$, Nano Lett. 19, 3138 (2019).

[4] K. S. Burch, D. Mandrus, and J.-G. Park, Magnetism in Two-Dimensional van der Waals Materials, Nature (London) 563, 47 (2018).

[5] M. Gibertini, M. Koperski, A. F. Morpurgo, and K. S. Novoselov, Magnetic 2D Materials and Heterostructures, Nat. Nanotechnol. 14, 408 (2019).

[6] M. A. McGuire, H. Dixit, V. R. Cooper, and B. C. Sales, Coupling of Crystal Structure and Magnetism in the Layered, Ferromagnetic Insulator $\mathrm{CrI}_{3}$, Chem. Mater. 27, 612 (2015).

[7] M. A. Mcgurie, Crystal and Magnetic Structures in Layered, Transition Metal Dihalides and Trihalides, Crystal 7, 121 (2017). 
[8] J. Kanamori, Superexchange Interaction and Symmetry Properties of Electron Orbitals, J. Phys. Chem. Solids 10, 87 (1959).

[9] D.-H. Kim, K. Kim, K.-T. Ko, J. H. Seo, J. S. Kim, T.-H. Jang, Y. Kim, J.-Y. Kim, S.-W. Cheong, and J.-H. Park, Giant Magnetic Anisotropy Induced by Ligand LS Coupling in Layered Cr Compounds, Phys. Rev. Lett. 122, 207201 (2019).

[10] A. T. Costa, D. L. R. Santos, N. M. R. Peres, and J. Fernández-Rossier, Topological Magnons in $\mathrm{CrI}_{3}$ Monolayers: An Itinerant Fermion Description, 2D Mater. 7, 045031 (2020).

[11] N. D. Mermin and H. Wagner, Absence of Ferromagnetism or Antiferromagnetism in One- or Two-Dimensional Isotropic Heisenberg Models, Phys. Rev. Lett. 17, 1133 (1966).

[12] A. Kartsev, M. Augustin, R. F. L. Evans, K. S. Novoselov, and E. J. G. Santos, Biquadratic Exchange Interactions in Two-Dimensional Magnets, npj Comput. Mater. 6, 150 (2020).

[13] M. Augustin, S. Jenkins, R. F. L. Evans, K. S. Novoselov, and E. J. G. Santos, Properties and Dynamics of Meron Topological Spin Textures in the Two-Dimensional Magnet $\mathrm{CrCl}_{3}$, Nat. Commun. 12, 185 (2021).

[14] D. A. Wahab et al., Quantum Rescaling, Domain Metastability, and Hybrid Domain-Walls in $2 \mathrm{D} \mathrm{CrI}_{3}$ Magnets, Adv. Mater. 33, 2004138 (2021).

[15] A. Y. Kitaev, Anyons in an Exactly Solved Model and Beyond, Ann. Phys. (Amsterdam) 321, 2 (2006).

[16] G. Jackeli and G. Khaliullin, Mott Insulators in the Strong Spin-Orbit Coupling Limit: From Heisenberg to a Quantum Compass and Kitaev Models, Phys. Rev. Lett. 102, 017205 (2009).

[17] H. Takagi, T. Takayama, G. Jackeli, G. Khaliullin, and S. E. Nagler, Concept and Realization of Kitaev Quantum Spin Liquids, Nat. Rev. Phys. 1, 264 (2019).

[18] C. Xu, J. Feng, H. Xiang, and L. Bellaiche, Interplay between Kitaev Interaction and Single Ion Anisotropy in Ferromagnetic $\mathrm{CrI}_{3}$ and $\mathrm{CrGeTe}_{3}$ Monolayers, npj Comput. Mater. 4, 57 (2018).

[19] I. Lee, F. G. Utermohlen, D. Weber, K. Hwang, C. Zhang, J. van Tol, J. E. Goldberger, N. Trivedi, and P. C. Hammel, Fundamental Spin Interactions Underlying the Magnetic Anisotropy in the Kitaev Ferromagnet $\mathrm{CrI}_{3}$, Phys. Rev. Lett. 124, 017201 (2020).

[20] P. P. Stavropoulos, X. Liu, and H.-Y. Kee, Magnetic Anisotropy in Spin-3/2 with Heavy Ligand in Honeycomb Mott Insulators: Application to $\mathrm{CrI}_{3}$, Phys. Rev. Research 3, 013216 (2021).

[21] D. G. Joshi, Topological Excitations in the Ferromagnetic Kitaev-Heisenberg Model, Phys. Rev. B 98, 060405(R) (2018).

[22] E. Aguilera, R. Jaeschke-Ubiergo, N. Vidal-Silva, L. E. F. F. Torres, and A.S. Nunez, Topological Magnonics in the Two-Dimensional van der Waals Magnet $\mathrm{CrI}_{3}$, Phys. Rev. B 102, 024409 (2020).

[23] S. A. Owerre, A First Theoretical Realization of Honeycomb Topological Magnon Insulator, J. Phys. Condens. Matter 28, 386001 (2016).
[24] S. K. Kim, H. Ochoa, R. Zarzuela, and Y. Tserkovnyak, Realization of the Haldane-Kane-Mele Model in a System of Localized Spins, Phys. Rev. Lett. 117, 227201 (2016).

[25] A. H. C. Neto, F. Guinea, N. M. R. Peres, K. S. Novoselov, and A. K. Geim, The Electronic Properties of Graphene, Rev. Mod. Phys. 81, 109 (2009).

[26] F. D. M. Haldane, Model for a Quantum Hall Effect without Landau Levels: Condensed-Matter Realization of the “Parity Anomaly", Phys. Rev. Lett. 61, 2015 (1988).

[27] A. V.Chumak, V. I. Vasyuchka, A. A. Serga, and B. Hillebrands, Magnon Spintronics, Nat. Phys. 11, 453 (2015).

[28] A. L. Chernyshev and P. A. Maksimov, Damped Topological Magnons in the Kagome-Lattice Ferromagnets, Phys. Rev. Lett. 117, 187203 (2016).

[29] X. S. Wang, Y. Su, and X. R. Wang, Topologically Protected Unidirectional Edge Spin Waves and Beam Splitter, Phys. Rev. B 95, 014435 (2017).

[30] L. Chen, J.-H. Chung, B. Gao, T. Chen, M. B. Stone, A. I. Kolesnikov, Q. Huang, and P. Dai, Topological Spin Excitations in Honeycomb Ferromagnet $\mathrm{CrI}_{3}$, Phys. Rev. X 8, 041028 (2018).

[31] I. Dzyaloshinskii, A Thermodynamic Theory of "Weak" Ferromagnetism of Antiferromagnetics, J. Phys. Chem. Solids 4, 241 (1958).

[32] T. Moriya, Anisotropic Superexchange Interaction and Weak Ferromagnetism, Phys. Rev. 120, 91 (1960).

[33] L. Chen et al., Magnetic Anisotropy in Ferromagnetic $\mathrm{CrI}_{3}$, Phys. Rev. B 101, 134418 (2020).

[34] L. Ke and M. I. Katsnelson, Electron Correlation Effects on Exchange Interactions and Spin Excitations in $2 D$ van der Waals Materials, npj Comput. Mater. 7, 4 (2021).

[35] X.-X. Zhang, L. Li, D. Weber, J. Goldberger, K. F. Mak, and J. Shan, Gate-Tunable Spin Waves in Antiferromagnetic Atomic Bilayers, Nat. Mater. 19, 838 (2020).

[36] J. Cenker et al., Direct Observation of Two-Dimensional Magnons in Atomically Thin $\mathrm{CrI}_{3}$, Nat. Phys. 17, 20 (2021).

[37] T. Li et al., Pressure-Controlled Interlayer Magnetism in Atomically Thin $\mathrm{CrI}_{3}$, Nat. Mater. 18, 1303 (2019).

[38] T. Song et al., Switching 2D Magnetic States via Pressure Tuning of Layer Stacking, Nat. Mater. 18, 1298 (2019).

[39] S. Li et al., Magnetic-Field-Induced Quantum Phase Transitions in a van der Waals Magnet, Phys. Rev. X 10, 011075 (2020).

[40] G. E. Granroth, A. I. Kolesnikov, T. E. Sherline, J. P. Clancy, K. A. Ross, J. P. C. Ruff, B. D. Gaulin, and S. E. Nagler, SEQUOIA: A Newly Operating Chopper Spectrometer at the SNS, J. Phys. Conf. Ser. 251, 012058 (2010).

[41] B. Winn et al., Recent Progress on HYSPEC, and Its Polarization Analysis Capabilities, EPJ Web Conf. 83, 03017 (2015).

[42] D. L. Abernathy, M. B. Stone, M. J. Loguillo, M. S. Lucas, O. Delaire, X. Tang, J. Y. Y. Lin, and B. Fultz, Design and Operation of the Wide Angular-Range Chopper Spectrometer ARCS at the Spallation Neutron Source, Rev. Sci. Instrum. 83, 015114 (2012).

[43] See Supplemental Material at http://link.aps.org/ supplemental/10.1103/PhysRevX.11.031047 for additional data and analysis.

[44] Y. O. Kvashnin, A. Bergman, A. I. Lichtenstein, and M. I. Katsnelson, Relativistic Exchange Interactions in $\mathrm{Cr} \mathrm{X}_{3}$ 
( $X=\mathrm{Cl}, \mathrm{Br}, \mathrm{I})$ Monolayers, Phys. Rev. B 102, 115162 (2020).

[45] A. Mook, K. Plekhanov, J. Klinovaja, and D. Loss, Interaction-Stabilized Topological Magnon Insulator in Ferromagnets, Phys. Rev. X 11, 021061 (2021).

[46] S. S. Pershoguba, S. Banerjee, J. C. Lashley, J. Park, H. A. gren, G. Aeppli, and A. V. Balatsky, Dirac Magnons in Honeycomb Ferromagnets, Phys. Rev. X 8, 011010 (2018).

[47] P. A. McClarty and J. G. Rau, Non-Hermitian Topology of Spontaneous Magnon Decay, Phys. Rev. B 100, 100405(R) (2019).

[48] B. Yuan, I. Khait, G.-J. Shu, F. C. Chou, M. B. Stone, J. P. Clancy, A. Paramekanti, and Y.-J. Kim, Dirac Magnons in a Honeycomb Lattice Quantum XY Magnet $\mathrm{CoTiO}_{3}$, Phys. Rev. X 10, 011062 (2020).

[49] M. Elliot, P. A. McClarty, D. Prabhakaran, R. D. Johnson, H. C. Walker, P. Manuel, and R. Coldea, Visualization of Isospin Momentum Texture of Dirac Magnons and Excitons in a Honeycomb Quantum Magnet, arXiv:2007.04199v1.

[50] R. Chisnell, J. S. Helton, D. E. Freedman, D. K. Singh, R. I. Bewley, D. G. Nocera, and Y. S. Lee, Topological Magnon Bands in a Kagome Lattice Ferromagnet, Phys. Rev. Lett. 115, 147201 (2015).

[51] R. Chisnell, J. S. Helton, D. E. Freedman, D. K. Singh, F. Demmel, C. Stock, D. G. Nocera, and Y. S. Lee, Magnetic Transitions in the Topological Magnon Insulator Cu(1,3-bdc), Phys. Rev. B 93, 214403 (2016).

[52] Z. Wang, I. Gutiérrez-Lezama, N. Ubrig, M. Kroner, M. Gibertini, T. Taniguchi, K. Watanabe, A. Imamolu, E. Giannini, and A.F. Morpurgo, Very Large Tunneling Magnetoresistance in Layered Magnetic Semiconductor $\mathrm{CrI}_{3}$, Nat. Commun. 9, 2516 (2018).

[53] F. Cantos-Prieto, A. Falin, M. Alliati, D. Qian, R. Zhang, T. Tao, M. R. Barnett, E. J. G. Santos, L. H. Li, and E. Navarro-Moratalla, Layer-Dependent Mechanical Properties and Enhanced Plasticity in the van der Waals Chromium Trihalide Magnets, Nano Lett. 21, 3379 (2021).
[54] C. de la Cruz et al., Magnetic Order Close to Superconductivity in the Iron-Based Layered $\mathrm{LaO}_{1-x} \mathrm{~F}_{x} \mathrm{FeAs}$ Systems, Nature (London) 453, 899 (2008).

[55] J. Zhao, W. Ratcliff II, J. W. Lynn, G. F. Chen, J. L. Luo, N. L. Wang, J. Hu, and P. Dai, Spin and Lattice Structures of Single-Crystalline $\mathrm{SrFe}_{2} \mathrm{As}_{2}$, Phys. Rev. B 78, 140504(R) (2008).

[56] J. Meseguer-Sanchez, D. A. Wahab, H. Luetkens, G. Taniashvili, E. Navarro-Moratalla, Z. Guguchia, and E. J. G. Santos, Thermal Disorder Driven Magnetic Phases in van der Waals Magnet $\mathrm{CrI}_{3}$, arXiv:2010.04544.

[57] P. Jiang, C. Wang, D. Chen, Z. Zhong, Z. Yuan, Z.-Y. Lu, and W. Ji, Stacking Tunable Interlayer Magnetism in Bilayer $\mathrm{CrI}_{3}$, Phys. Rev. B 99, 144401 (2019).

[58] J. Shang, X. Tang, X. Tan, A. Du, T. Liao, S. C. Smith, Y. Gu, C. Li, and L. Kou, Stacking-Dependent Interlayer Magnetic Coupling in $2 \mathrm{D} \quad \mathrm{CrI}_{3} / \mathrm{CrGeTe}_{3}$ Nanostructures for Spintronics, ACS Appl. Nano Mater. 3, 1282 (2020).

[59] S. W. Jang, M. Y. Jeong, H. Yoon, S. Ryee, and M. J. Han, Microscopic Understanding of Magnetic Interactions in Bilayer $\mathrm{CrI}_{3}$, Phys. Rev. Mater. 3, 031001(R) (2019).

[60] B. Niu et al., Coexistence of Magnetic Orders in TwoDimensional Magnet $\mathrm{CrI}_{3}$, Nano Lett. 20, 553 (2020).

[61] B. Huang, J. Cenker, X. Zhang, E. L. Ray, T. Song, T. Taniguchi, K. Watanabe, M. A. McGuire, D. Xiao, and X. $\mathrm{Xu}$, Tuning Inelastic Light Scattering via Symmetry Control in the Two-Dimensional Magnet $\mathrm{CrI}_{3}$, Nat. Nanotechnol. 15, 212 (2020).

[62] Z. Sun et al., Giant Nonreciprocal Second-Harmonic Generation from Antiferromagnetic Bilayer $\mathrm{CrI}_{3}$, Nature (London) 572, 497 (2019).

[63] A. McCreary et al., Distinct Magneto-Raman Signatures of Spin-Flip Phase Transitions in $\mathrm{CrI}_{3}$, Nat. Commun. 11, 3879 (2020). 